Article

\title{
Synthesis, Reactions and Biological Evaluation of Some New Naphtho[2,1-b]furan Derivatives Bearing a Pyrazole Nucleus
}

\author{
Ashraf H. F. Abd El-Wahab ${ }^{1, *}$, Zarrag Isa A. Al-Fifi ${ }^{2}$, Ahmed H. Bedair ${ }^{3}$, Fawzy M. Ali ${ }^{3}$, \\ Ahmed H. A. Halawa ${ }^{3}$ and Ahemed M. El-Agrody ${ }^{3}$ \\ 1 Department of Chemistry, Faculty of Science, Jazan University, Jazan 2097, Saudi Arabia \\ 2 Department of Biology, Faculty of Science, Jazan University, Jazan 2097, Saudi Arabia \\ 3 Department of Chemistry, Faculty of Science, Al-Azhar University, Nasr City 11884, Cairo, Egypt \\ * Author to whom correspondence should be addressed; E-Mail: ash_abdelwahab@yahoo.com; \\ Tel.: 00960540963753; Fax: 0096073245212.
}

Received: 14 November 2010; in revised form: 14 December 2010 / Accepted: 15 December 2010 / Published: 5 January 2011

\begin{abstract}
Vilsmeier formylation of 2-(1-phenylhydrazonoethyl)naphtho[2,1-b]furan (2) gave 3-naphtho[2,1-b]furan-2-yl-1-phenyl-1H-pyrazole-4-carbaldehyde (3), which was reacted with $\mathrm{C}$ - and $\mathrm{N}$-nucleophiles to afford naphthofuranpyrazol derivatives 4-8. Treatment of 2-[(3-(naphtho[2,1-b]furan-2-yl)-1-phenyl-1H-pyrazol-4-yl)methylene]malononitrile (4a) with reactants having active hydrogen and $\mathrm{Et}_{3} \mathrm{~N}$ gave the corresponding pyrazoline, pyran and chromene addition product derivatives 10, 12 and 13, consisting of three different connected heterocyclic moieties. Reaction of 1-((3-(naphtho[2,1-b]furan-2yl)-1-phenyl-1H-pyrazol-4-yl) methylene)-2-phenylhydrazone (6b) with AcONa and ethyl bromoacetate or chloroacetone afforded the thiazolidinone and methylthiazole derivatives 14 and 15, respectively. In addition, intramolecular cyclization of $\mathbf{6 d}$ with $\mathrm{Ac}_{2} \mathrm{O}$ afford the corresponding 1,3,4-thiadiazol-2-yl acetamide derivative 16. The structures of the synthesized compounds were confirmed by IR, ${ }^{1} \mathrm{H}-\mathrm{NMR} /{ }^{13} \mathrm{C}-\mathrm{NMR}$ and mass spectral studies. Compound $\mathbf{1 4}$ showed promising effects against the tested Gram positive and negative bacteria and fungi.
\end{abstract}

Keywords: Vilsmeier reaction; 2-[3-(naphtho[2,1-b]furan-2-yl)-1-phenyl-1H-pyrazol-4yl)methylene]malononitrile; 1,3,4-thiadiazol-2-ylacetamide; antimicrobial activity 


\section{Introduction}

Naphthofuran derivatives exhibit very potent antibacterial [1-3], genotoxic [4-6] and anticancer activity [7-9], but some were shown to be mutagenic in bacteria [10-15]. The present investigation deals with the synthesis of some new naphtho[2,1-b]furan derivatives bearing pyrazole nuclei attached to different heterocyclic moieties and their biological activities.

\section{Results and Discussion}

Condensation of 2-acetylnaphtho[2,1-b]furan (1) [16] with phenylhydrazine afforded 2-(1phenylhydrazonoethyl)naphtho[2,1-b]furan (2). Vilsmeier formylation [17] of the latter afforded 3(naphtho-[2,1-b]furan-2-yl)-1-phenyl-1H-pyrazole-4-carboxaldehyde (3). Condensation of 3 with a variety of C-nucleophiles, namely malononitrile, cyanoacetamide, cyanothioacetamide, barbituric acid and 2-acetylnaphtho[2,1-b]furan (1) give the condensation products $\mathbf{4}, \mathbf{5}$ and $\mathbf{8}$, while reactions with $\mathrm{N}$ nucleophiles, namely hydrazine derivatives or amines, afforded the condensation products $\mathbf{6}$ and $\mathbf{7}$ (Scheme 1).

Scheme 1. Synthesis of naphtho[2,1-b]furan pyrazole derivatives.

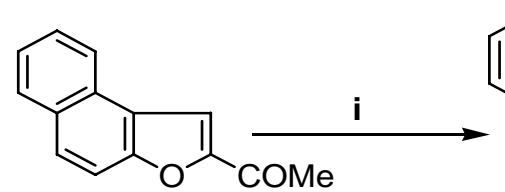<smiles>C/C(=N\Nc1ccccc1)c1cc2c3c(ccc2o1)-c1ccccc1-3</smiles>

1

2<smiles>[R]c1nn(-c2ccccc2)cc1C=C1C(=O)NC(=O)NC1=O</smiles><smiles>[R]c1nn(-c2ccccc2)cc1C=O</smiles>

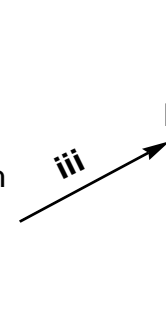

3<smiles>[R]OC=Cc1cn(-c2ccccc2)nc1[R]</smiles>

8<smiles></smiles>

$\mathrm{R}=$<smiles>[R]c1nn(-c2ccccc2)cc1C=[W]</smiles>

7a; $\mathrm{Ar}=\mathrm{C}_{6} \mathrm{H}_{5}$

b; $\mathrm{Ar}=\mathrm{C}_{6} \mathrm{H}_{4} \mathrm{Cl}-\mathrm{p}$

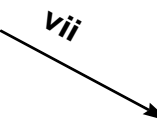

CH=NNHR

6a; $\mathrm{R}^{\prime}=\mathrm{H}$

b; $\mathrm{R}^{\prime}=\mathrm{Ph}$

c; $\mathrm{R}^{\prime}=\mathrm{COMe}$

d; $\mathrm{R}^{\prime}=\mathrm{CSNH}_{2}$

e; $\mathrm{R}^{\prime}=\mathrm{COCH}_{2} \mathrm{CN}$

4a; $X=C N$

b; $\mathrm{X}=\mathrm{CONH}_{2}$

c; $\mathrm{X}=\mathrm{CSNH}_{2}$

$$
\text { se }
$$

EtOH, piperidine, reflux; iv) barbaturic acid, EtOH, triethylamine, reflux; v) 2-acetylnaphtho[2,1b]furan (1); vi) aniline or $p$-chloroaniline, EtOH, reflux; vii) hydrazine derivatives, EtOH, reflux. 
Compounds 4a and $\mathbf{6 d}$ were used as key intermediates in the synthesis of novel pyran, pyrazole and thiazole derivatives via their interaction with different reagents. Thus, the reaction of 4a with 3methyl-1-phenyl-2-pyrazolin-5-one in the presence of triethylamine did not give the expected pyrazolopyran 9 and instead, only one compound was isolated, identified as 4-[3-(naphtho[2,1-b]furan2-yl)-1-phenyl-1H-pyrazol-4-yl]methylene-3-methyl-1-phenyl-2-pyrazoline-5-one (10), while with hydrazine hydrate in refluxing ethanol it furnished 6a (as verified by m.p. and mixed m.p.) instead of the pyrazole derivative 11. The formation of $\mathbf{6 a}$ and $\mathbf{1 0}$ were assumed to proceed via elimination of malononitrile [18] and their structures were further confirmed by independent synthesis via direct condensation of 3 with hydrazine hydrate (Scheme 1) or with 3-methyl-1-phenyl-2-pyrazolin-5-one (Scheme 2), respectively The reaction of 4 a with ethyl acetoacetate in dry methylene chloride containing triethylamine gave the pyrazolopyran derivative 12, while with 4-hydroxycoumarin under Michael reaction conditions it afforded the 4H-pyran derivative 13 (Scheme 2). The structure of 13 was further verified by m.p. and mixed m.p after independent synthesis via direct condensation of 3 with 4-hydroxycoumarin in the presence of malononitrile and a few drops of piperidine as a base (a one pot reaction).

Scheme 2. Synthesis of pyran, pyrazole and thiazole derivatives.
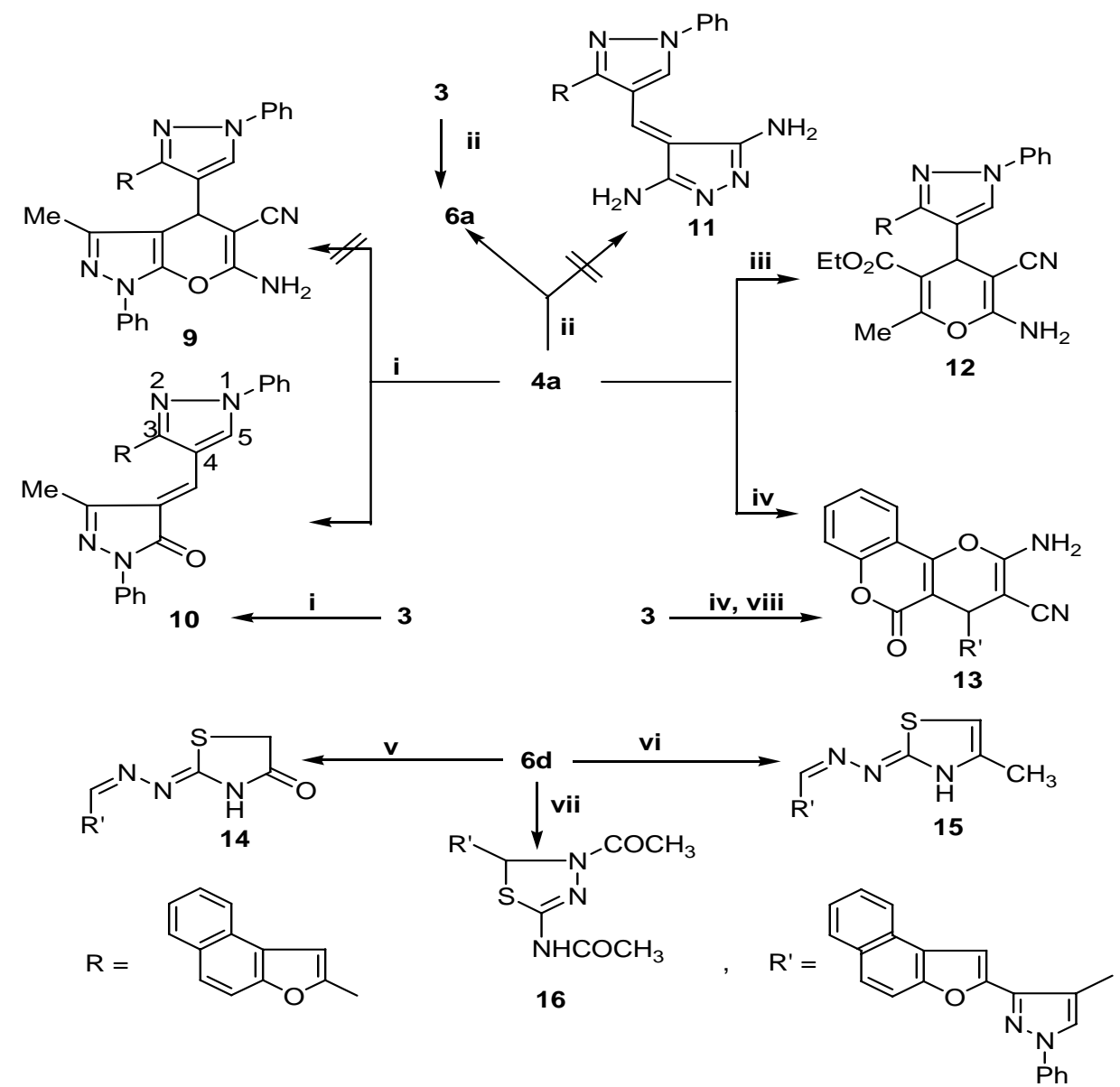

Reagents and Conditions: i) 3-methyl-1-phenyl-2-pyrazolin-5-one, EtOH, triethylamine, reflux; ii) $\mathrm{NH}_{2} \mathrm{NH}_{2} \cdot \mathrm{H}_{2} \mathrm{O}$, EtOH, reflux; iii) $\mathrm{AcCH}_{2} \mathrm{CO}_{2} \mathrm{Et}, \mathrm{CH}_{2} \mathrm{Cl}_{2}$, triethylamine, reflux; iv) 4-hydroxycoumarin, EtOH, piperidine, reflux; v) $\mathrm{BrCH}_{2} \mathrm{CO}_{2} \mathrm{Et}, \mathrm{CH}_{3} \mathrm{CO}_{2} \mathrm{Na}$, EtOH, reflux; vi) $\mathrm{ClCH}_{2} \mathrm{COCH}_{3}$, $\mathrm{CH}_{3} \mathrm{CO}_{2} \mathrm{Na}$, EtOH, reflux; vii) $\mathrm{Ac}_{2} \mathrm{O}$, reflux, viii) $\mathrm{CH}_{2}(\mathrm{CN})_{2}$. 
When thiosemicarbazone derivative 6d was allowed to react with ethyl bromoacetate or chloroacetone in the presence of fused sodium acetate, it gave the corresponding thiazolidinone and thiazoline derivatives 14 and 15, respectively, while with refluxing acetic anhydride it afforded the thiadiazole derivative 16 (Scheme 2). These reactions were assumed to proceed via $S$-alkylation followed by intramolecular cyclization with concomitant loss of an alcohol molecule and/or dehydration.

Structures 2, 3, 4, 5, 6, 7 and 8 were established by spectral and physical data (Tables I-II). The mass spectra of 3, 4b,c, 5, 6a-d, 7a,b and $\mathbf{8}$ showed the corresponding molecular ion peaks at $\mathrm{m} / \mathrm{z} 338$ $\left(\mathrm{M}^{+}, 100 \%\right), \mathrm{m} / \mathrm{z} 420\left(\mathrm{M}^{+}, 35 \%\right), \mathrm{m} / \mathrm{z} 404\left(\mathrm{M}^{+}, 100 \%\right), \mathrm{m} / \mathrm{z} 448\left(\mathrm{M}^{+}, 100 \%\right), \mathrm{m} / \mathrm{z} 352\left(\mathrm{M}^{+}, 100 \%\right)$, m/z $404\left(\mathrm{M}^{+}, 100 \%\right), \mathrm{m} / \mathrm{z} 394\left(\mathrm{M}^{+}, 100 \%\right), \mathrm{m} / \mathrm{z} 411\left(\mathrm{M}^{+}, 2 \%\right), \mathrm{m} / \mathrm{z} 413\left(\mathrm{M}^{+}, 100 \%\right), \mathrm{m} / \mathrm{z} 447\left(\mathrm{M}^{+}\right.$, $100 \%)$ and $\mathrm{m} / \mathrm{z} 530\left(\mathrm{M}^{+}, 65 \%\right)$, respectively. The fragmentation patterns of compounds 3, 4b,c, 5, 6ad, $7 \mathbf{a}, \mathbf{b}$ and 8 are illustrated in Scheme 3.

Table I. Physical and analytical data of new synthesized compounds 1-16.

\begin{tabular}{|c|c|c|c|c|c|}
\hline \multirow{2}{*}{$\begin{array}{l}\text { Comp. } \\
\text { No. }\end{array}$} & \multirow{2}{*}{$\begin{array}{l}\text { M.P. } \\
\left(\mathrm{T} /{ }^{0} \mathrm{C}\right)^{\mathrm{a}}\end{array}$} & \multirow{2}{*}{$\begin{array}{c}\text { Molecular } \\
\text { Formula (MW) }\end{array}$} & \multicolumn{3}{|c|}{$\begin{array}{c}\text { Analysis (\%) } \\
\text { Found/calculated }\end{array}$} \\
\hline & & & C & $\mathbf{H}$ & $\mathbf{N}$ \\
\hline 1 & $108-110^{a}$ & $\mathrm{C}_{14} \mathrm{H}_{10} \mathrm{O}_{2}(210.23)$ & $79.95(79.98)$ & $4.78(4.79)$ & - \\
\hline 2 & $172-174^{\mathrm{a}}$ & $\mathrm{C}_{20} \mathrm{H}_{16} \mathrm{~N}_{2} \mathrm{O}(300.35)$ & $79.96(79.98)$ & $5.35(5.37)$ & $9.30(9.33)$ \\
\hline 3 & $180-182^{\mathrm{a}}$ & $\mathrm{C}_{22} \mathrm{H}_{14} \mathrm{~N}_{2} \mathrm{O}_{2}(338.36)$ & $78.00(78.09)$ & $4.12(4.17)$ & $8.20(8.28)$ \\
\hline $4 a$ & $185-187^{\mathrm{a}}$ & $\mathrm{C}_{25} \mathrm{H}_{14} \mathrm{~N}_{4} \mathrm{O}(386.40)$ & $77.65(77.71)$ & $3.60(3.65)$ & $14.47(14.50)$ \\
\hline $4 \mathbf{b}$ & $292-294^{\mathrm{b}}$ & $\mathrm{C}_{25} \mathrm{H}_{16} \mathrm{~N}_{4} \mathrm{O}_{2}(404.42)$ & $74.20(74.25)$ & $3.95(3.99)$ & $13.78(13.85)$ \\
\hline $4 c$ & $265-267^{\mathrm{a}}$ & $\mathrm{C}_{25} \mathrm{H}_{16} \mathrm{~N}_{4} \mathrm{OS}(420.49)$ & $71.35(71.41)$ & $3.80(3.84)$ & $13.25(13.32)$ \\
\hline 5 & $350-352^{\mathrm{a}}$ & $\mathrm{C}_{26} \mathrm{H}_{16} \mathrm{~N}_{4} \mathrm{O}_{4}(448.43)$ & $69.60(69.64)$ & $3.55(3.60)$ & $12.35(12.49)$ \\
\hline $6 a$ & $280-282^{c}$ & $\mathrm{C}_{22} \mathrm{H}_{16} \mathrm{~N}_{4} \mathrm{O}(352.39)$ & $74.90(74.98)$ & $4.50(4.58)$ & $15.85(15.90)$ \\
\hline $6 \mathbf{b}$ & $190-192^{\mathrm{d}}$ & $\mathrm{C}_{29} \mathrm{H}_{24} \mathrm{~N}_{4} \mathrm{O}(444.53)$ & $78.29(78.36)$ & $5.35(5.44)$ & $12.55(12.60)$ \\
\hline 6c & $220-222^{\mathrm{d}}$ & $\mathrm{C}_{25} \mathrm{H}_{22} \mathrm{~N}_{4} \mathrm{O}_{2}(410.47)$ & $73.10(73.15)$ & $5.32(5.40)$ & $13.60(13.65)$ \\
\hline 6d & $228-230^{\mathrm{b}}$ & $\mathrm{C}_{23} \mathrm{H}_{17} \mathrm{~N}_{5} \mathrm{OS}(411.48)$ & $67.09(67.13)$ & $4.12(4.16)$ & $16.95(17.02)$ \\
\hline $6 e$ & $345-347^{\mathrm{e}}$ & $\mathrm{C}_{25} \mathrm{H}_{17} \mathrm{~N}_{5} \mathrm{O}_{2}(419.43)$ & $71.50(71.59)$ & $4.05(4.09)$ & $16.67(16.70)$ \\
\hline $7 a$ & $174-176^{\mathrm{d}}$ & $\mathrm{C}_{29} \mathrm{H}_{23} \mathrm{~N}_{3} \mathrm{O}(429.51)$ & $81.00(81.09)$ & $5.32(5.40)$ & $9.70(9.78)$ \\
\hline $7 \mathbf{b}$ & $170-172^{\mathrm{d}}$ & $\mathrm{C}_{29} \mathrm{H}_{22} \mathrm{ClN}_{3} \mathrm{O}(463.96)$ & $75.00(75.07)$ & $4.70(4.78)$ & $9.00(9.06)$ \\
\hline 8 & $260-262^{b}$ & $\mathrm{C}_{36} \mathrm{H}_{22} \mathrm{~N}_{2} \mathrm{O}_{3}(530.57)$ & $81.40(81.49)$ & $4.12(4.18)$ & $5.25(5.28)$ \\
\hline 10 & $218-220^{c}$ & $\mathrm{C}_{32} \mathrm{H}_{22} \mathrm{~N}_{4} \mathrm{O}_{2}(494.54)$ & $77.70(77.72)$ & $4.35(4.48)$ & $11.30(11.33)$ \\
\hline 12 & $208-210^{\mathrm{a}}$ & $\mathrm{C}_{31} \mathrm{H}_{24} \mathrm{~N}_{4} \mathrm{O}_{4}(516.55)$ & $72.00(72.08)$ & $4.60(4.68)$ & $10.80(10.85)$ \\
\hline 13 & $260-262^{b}$ & $\mathrm{C}_{34} \mathrm{H}_{20} \mathrm{~N}_{44} \mathrm{O}_{4}(548.55)$ & $74.40(74.44)$ & $3.60(3.67)$ & $10.18(10.21)$ \\
\hline 14 & $271-273^{c}$ & $\mathrm{C}_{25} \mathrm{H}_{17} \mathrm{~N}_{5} \mathrm{O}_{2} \mathrm{~S}(451.50)$ & $66.45(66.50)$ & $3.72(3.80)$ & $15.45(15.51)$ \\
\hline 15 & $220-222^{c}$ & $\mathrm{C}_{26} \mathrm{H}_{19} \mathrm{~N}_{5} \mathrm{OS}(449.53)$ & $69.42(69.47)$ & $4.23(4.26)$ & $15.54(15.85)$ \\
\hline 16 & $255-257^{\mathrm{c}}$ & $\mathrm{C}_{27} \mathrm{H}_{21} \mathrm{~N}_{5} \mathrm{O}_{3} \mathrm{~S}(495.55)$ & $65.38(65.44)$ & $4.23(4.27)$ & $14.00(14.13)$ \\
\hline
\end{tabular}

${ }^{\mathrm{a}}$ From EtOH; ${ }^{\mathrm{b}}$ from dioxane; ${ }^{\mathrm{c}}$ from acetic acid; ${ }^{\mathrm{d}}$ from benzene; ${ }^{\mathrm{e}}$ from DMF 
Table II. Significant peaks in the EI (70 ev) spectra of compounds 1, 3, 4b,c, 5, 6a-d,7b, $8,10,12,13,16$.

\begin{tabular}{|c|c|c|c|c|c|c|c|c|}
\hline \multirow{2}{*}{$\begin{array}{l}\text { Comp. } \\
\text { No. }\end{array}$} & \multicolumn{8}{|c|}{$m / z$ (intensity/\%) } \\
\hline & $\mathbf{M}^{+}$ & 336 & 310 & 232 & 193 & 139 & 77 & Other peaks \\
\hline 1 & $210(32)$ & & & & & $139(100)$ & & $\begin{array}{l}\text { 168(3), 113(11), 89(15), } \\
63(38) .\end{array}$ \\
\hline 3 & $338(100)$ & & $310(20)$ & & & $139(15)$ & $77(62)$ & $234(1), 208(1), 167(1)$ \\
\hline $\mathbf{4 b}$ & $404(100)$ & & & $232(1)$ & $193(3)$ & $139(12)$ & $77(67)$ & $360(25), 283(4), 257(3)$, \\
\hline $4 c$ & $420(35)$ & & $310(1)$ & & $193(8)$ & $139(15)$ & $77(58)$ & $386(100), 281(2)$ \\
\hline 5 & $448(100)$ & & & & & $139(7)$ & $77(34)$ & $\begin{array}{l}338(14), 290(11), 22 \\
4(5), 181(3)\end{array}$ \\
\hline 6a & $352(100)$ & $336(66)$ & & & & $139(30)$ & $77(44)$ & $168(20)$ \\
\hline $6 \mathbf{b}$ & $428(100)$ & $336(60)$ & & & & $139(13)$ & $77(64)$ & $351(1), 284(15), 176(11)$ \\
\hline 6c & $394(100)$ & $336(29)$ & & & & $139(5)$ & $77(20)$ & $\begin{array}{l}352(10), 309(2), 232(3), \\
180(1)\end{array}$ \\
\hline 6d & $411(1)$ & $336(100)$ & & & & & $77(32)$ & $\begin{array}{l}308(2), 233(2), 20(1), \\
181(1)\end{array}$ \\
\hline $7 \mathbf{b}$ & $530(65)$ & $336(100)$ & & $232(5)$ & & $139(41)$ & $77(28)$ & $258(4), 195(16)$ \\
\hline 8 & $447(100)$ & $336(7)$ & $310(2)$ & & & & & $\begin{array}{l}233(3), 207(5), 141(15), \\
76(64)\end{array}$ \\
\hline 10 & $494(100)$ & & $310(2)$ & & $193(5)$ & $139(8)$ & $77(31)$ & $466(2), 386(25), 233(4)$ \\
\hline 12 & $516(2)$ & & & & $193(13)$ & $139(5)$ & $77(16)$ & $\begin{array}{l}386(100), 323(12), 254 \\
(4),\end{array}$ \\
\hline 13 & $548(2)$ & & $310(5)$ & & & $139(11)$ & & $\begin{array}{l}482(15), 414(9), 268 \\
(12), 215(25), 162(23) \\
71(60)\end{array}$ \\
\hline 16 & $495(45)$ & $336(100)$ & & $232(3)$ & 193(4) & & $77(40)$ & $453(18), 394(29), 306(4)$ \\
\hline
\end{tabular}

Structures 10, 11, 12, 13, and 16 were established by spectral data (Tables I-II). The mass spectra of 10, 12, 13 and 15 showed molecular ion peaks at $\mathrm{m} / \mathrm{z} ; 494\left(\mathrm{M}^{+}, 100\right), \mathrm{m} / \mathrm{z} ; 516\left(\mathrm{M}^{+}, 2\right), \mathrm{m} / \mathrm{z} ; 548\left(\mathrm{M}^{+}\right.$, $2)$, and $m / z ; 495\left(\mathrm{M}^{+}, 45\right)$, respectively. The fragmentation pattern of compounds $\mathbf{1 0}, \mathbf{1 2}, \mathbf{1 3}$ and 15 are illustrated in Scheme 3.

Scheme 3. Proposed fragmentation pathways for the EI spectra of the substituted naphtho[2,1-b]furans 3-16.
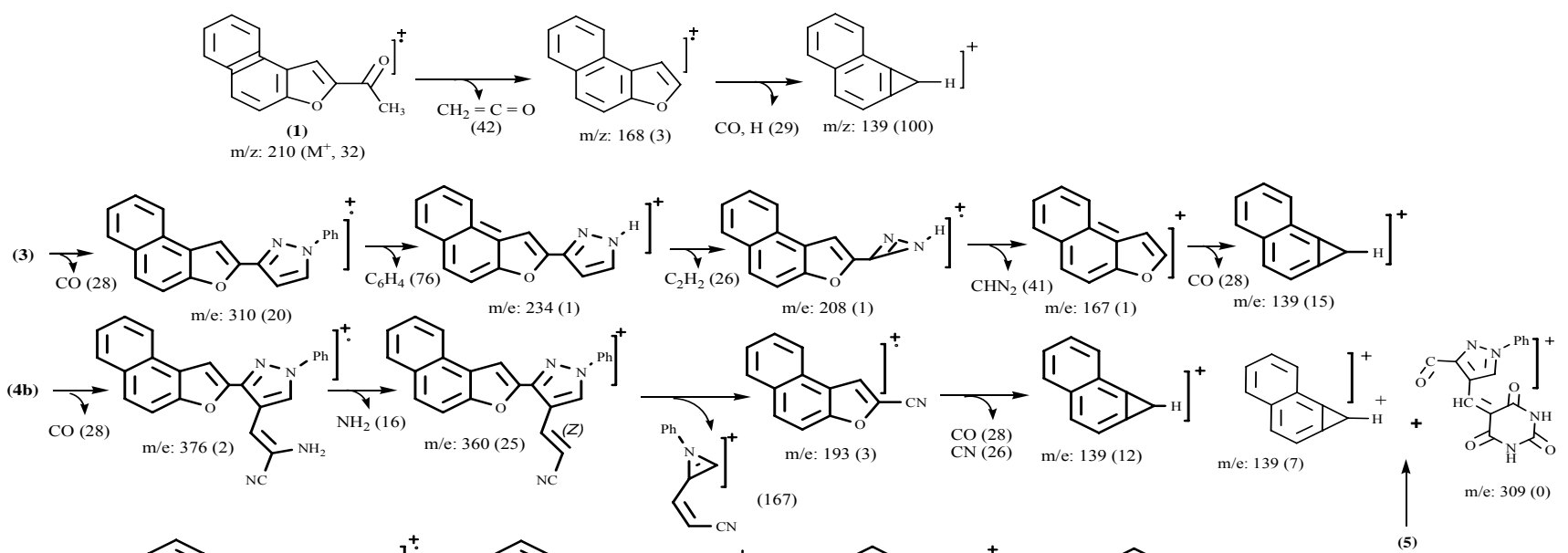<smiles>[CH2+]Cn1cc(C=C(C)C)c(-c2cc3c(ccc4ccccc43)o2)n1</smiles>

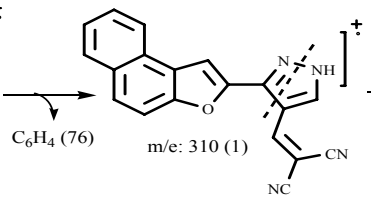<smiles></smiles>

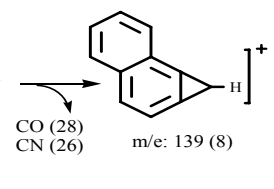


Scheme 3. Cont.

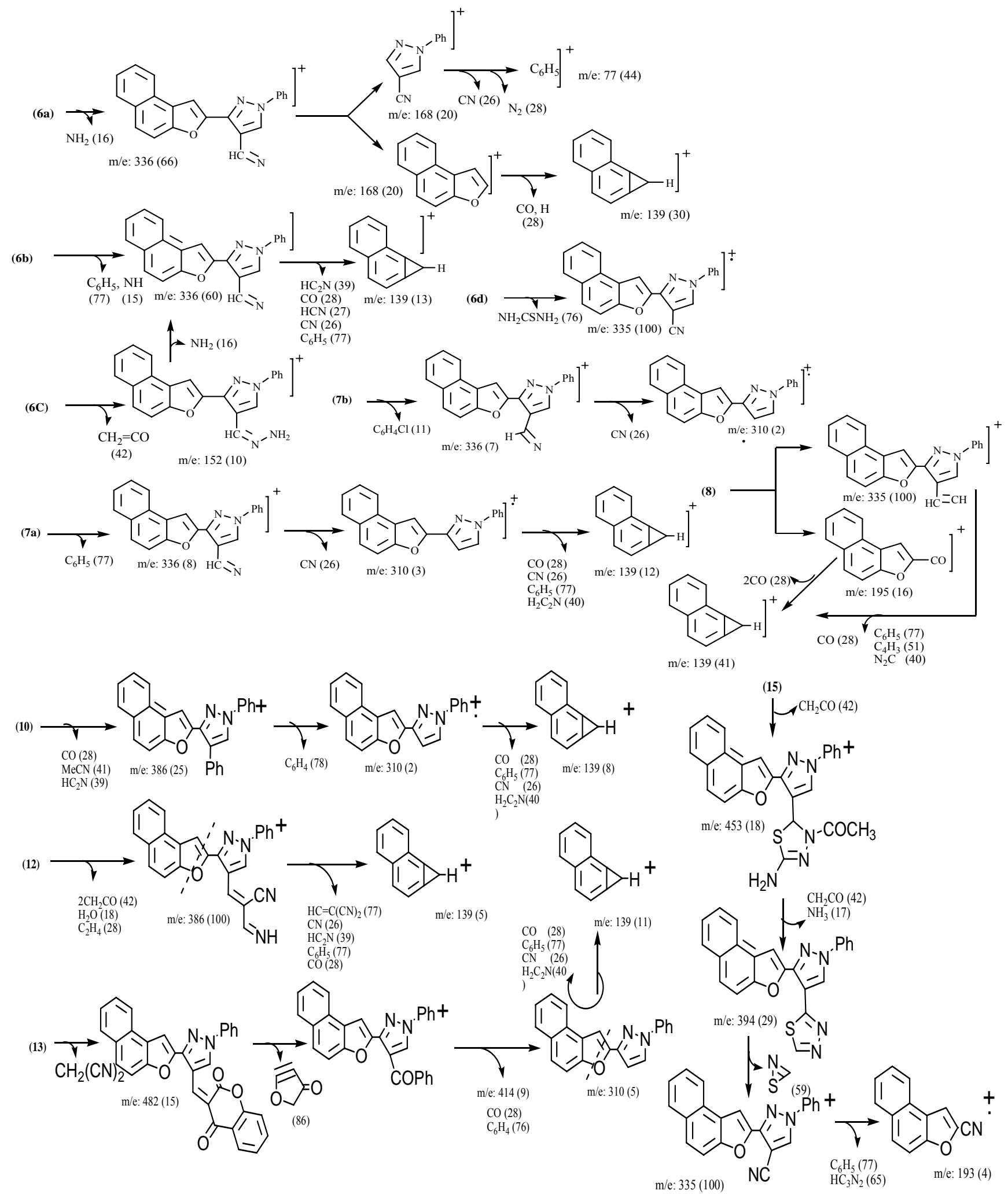

\section{Biological activities}

Compound 14 which contains the thiazolidin-4-one nucleus showed highest antibacterial activity (+++ ve inhibition zone was between $12-15 \mathrm{~mm}$ ) against Bacillus subtilis, Staphylococcus aureus, Escherichia coli, Pseudomonas aeuroginosa, Candida albicans and Aspergillus niger. Compound 2 
showed moderate inhibition (++ ve inhibition zone up to $8 \mathrm{~mm}$ ) against Bacillus subtilis, Staphylococcus aureus, Escherichia coli and Pseudomonas aeuroginosa, the remaining tested compounds 4a-c, 5, 6, 7a,b, 8a-c, 10, 12, 13 showed no activities against any of the test microorganisms.

\section{Experimental}

\section{General}

Melting points were measured on a Stuart Scientific Co (UK) melting point apparatus and are uncorrected. The IR spectra were recorded on a Shimadzu IR 440, spectrophotometer (Shimadzu, Japan) in $\mathrm{KBr}$. The ${ }^{1} \mathrm{H}-\mathrm{NMR} /{ }^{13} \mathrm{C}-\mathrm{NMR}$ spectra were measured on a Varian Mercury (300 MHz) spectrometer (Varian, UK), using TMS as an internal standard and DMSO- $\mathrm{d}_{6}$ as solvent. Mass spectra were run on a Shimadzu GC-Ms QP 1000 EX mass spectrometer. Microanalytical data were obtained from the Microanalytical Unit Center, Faculty of Science, Cairo University (Egypt). Spectral and microanalytical data are given in Tables I-III. The paper discs were manufactured by Bristol-Myers Squibb, Giza, Egypt.

Table III. Antibacterial and antifugal activities of select synthesized compounds.

\begin{tabular}{|c|c|c|c|c|c|c|}
\hline \multirow[b]{2}{*}{$\begin{array}{c}\text { Comp. } \\
\text { No. }\end{array}$} & \multicolumn{6}{|c|}{ Inhibition zone diameter (mm per 200 mcg sample) } \\
\hline & $\begin{array}{c}(B S) \\
\text { ACTC } \\
10400 \\
\end{array}$ & $\begin{array}{c}\text { (SA) } \\
\text { NCTC } \\
7447 \\
\end{array}$ & $\begin{array}{c}(E C) \\
\text { NCTC } \\
10410 \\
\end{array}$ & $\begin{array}{c}\text { (PA) } \\
\text { ATCC } \\
10415 \\
\end{array}$ & $\begin{array}{c}(C A) \\
\text { IMRU } \\
3669 \\
\end{array}$ & $\begin{array}{c}(A N) \\
\text { ATCC } \\
6275 \\
\end{array}$ \\
\hline 2 & $++\mathrm{ve}$ & $++\mathrm{ve}$ & $++\mathrm{ve}$ & $++\mathrm{ve}$ & - ve & $-\mathrm{ve}$ \\
\hline 14 & +++ ve & +++ ve & $+++\mathrm{ve}$ & +++ ve & +++ ve & $+++\mathrm{ve}$ \\
\hline $\begin{array}{l}\text { Neomycin } \\
\left(30 \mu \mathrm{g} \mathrm{mL}^{-1}\right)\end{array}$ & +++ ve & +++ ve & +++ ve & +++ ve & +++ ve & $+++\mathrm{ve}$ \\
\hline
\end{tabular}

Synthesis of 2-acetylnaphtho[2,1-b]furan (1): A mixture of 2-hydroxy-1-naphthaldehyde (0.01 mol), chloroacetone $(0.01 \mathrm{~mol})$ and anhydrous potassium carbonate $(0.02 \mathrm{~mol})$ in anhydrous acetone $(50$ $\mathrm{mL}$ ) was refluxed for 8 hours. The mixture was allowed to cool and poured onto crushed ice (50 gm) and water $(100 \mathrm{~mL})$ then acidified with conc. $\mathrm{HCl}$ and the solid product was formed was filtered off, washed with water and recrystallized from ethanol to give 1 (90\% yield); IR: 1,666 (CO): ${ }^{1} \mathrm{H}-\mathrm{NMR}$ : 8.59-7.70 (m, 6H, Ar-H), 7.89 (s, 1H, H-3), 2.62 (s, 3H, Me): ${ }^{13} \mathrm{C}-\mathrm{NMR}: 187.19$ (CO), 153.36 (C-9a), 151.90 (C-2), 130.11 (C-3a), 129.90 (C-8), 128.98 (C-7), 127.85 (C-7a), 127.52 (C-6), 125.60 (C-5), 123.64 (C-4), 122.61 (C-3b), 113.69 (C-3), 112.74 (C-9), 26.33 (Me).

Synthesis of 2-(1-phenylhydrazonoethyl)naphtho[2,1-b]furan (2): A mixture of 1 (1.72 g, $0.01 \mathrm{~mol})$ and phenylhydrazine $(1.08 \mathrm{~g}, 0.01 \mathrm{~mol})$ in ethanol $(50 \mathrm{~mL})$ was refluxed for $2 \mathrm{~h}$, the solid that separated on heating was filtered off and recrystallized from $\mathrm{EtOH}$ to give 2 (80\% yield); IR : 3,459, 3,346 $(\mathrm{NH}), 1,601(\mathrm{C}=\mathrm{N})$; ${ }^{1} \mathrm{H}-\mathrm{NMR}: 8.17-6.92(\mathrm{~m}, 12 \mathrm{H}, \mathrm{Ar}-\mathrm{H}+\mathrm{NH}), 7.70(\mathrm{~s}, 1 \mathrm{H}, \mathrm{H}-3), 2.35(\mathrm{~s}, 3 \mathrm{H}, \mathrm{Me}) ;{ }^{13} \mathrm{C}-$ NMR: 154.81 (C-9a), 151.72 (C=N), 145.45 (C-2), 132.94 (C-1 ), 130.01 (C-3a), 128.94 (C-8), 128.66 
(C-3`,5 '), 127.06 (C-7a), 126.44 (C-7), 125.28 (C-6), 124.75 (C-5), 123.97 (C-3b), 123.65 (C-4), 119.39 (C-4), 113.04 (C-3), 112.22 (C-9), 102.89 (C-2`,6'), 12.80 (Me).

Synthesis of 3-naphtho[2,1-b]furan-2-yl-1-phenyl-1H-pyrazole-4-carbaldehyde (3): To ice cold dimethylformamide $(0.01 \mathrm{~mol})$ was added dropwise with stirring phosphorus oxychloride $(0.01 \mathrm{~mol})$ over a period of $30 \mathrm{~min}$. stirring was continued for further $45 \mathrm{~min}$ keeping the reaction mixture at $0{ }^{\circ} \mathrm{C}$. The hydrazone 2 ( $3 \mathrm{~g}, 0.01 \mathrm{~mol})$ was then added and the reaction mixture was allowed to attain room temperature. The mixture was heated at $90{ }^{\circ} \mathrm{C}$ for $2 \mathrm{~h}$, allowed to cool and poured onto crushed ice (50 $\mathrm{gm})$ and water $(100 \mathrm{~mL})$, the solid product that was formed was filtered off and washed with water and recrystallized from EtOH to give 3 (70\% yield); IR: 1,670 (CO): ${ }^{1} \mathrm{H}-\mathrm{NMR}: 10.33$ (s, 1H, CHO), 9.37 (s, 1H, H-5), 8.43-7.47 (m, 11H, Ar-H), 7.92 (s, 1H, H-3').

Synthesis of 2-((3-(naphtho[2,1-b] furan-2-yl)-1-phenyl-1H-pyrazol-4-yl)methylene) malononitrile (4a), 2-cyano-3-(3-(naphtho[2,1-b] furan-2-yl)-1-phenyl-1H-pyrazol-4-yl) acrylamide (4b) and 2-cyano-3(3-(naphtho[2,1-b]furan-2-yl)-1-phenyl-1H-pyrazol-4-yl) prop-2-enethioamide (4c): A mixture of aldehyde 3 (3.38 g, $0.01 \mathrm{~mol})$ and malononitrile, cyanoacetamide or cyanothioacetamide $(0.01 \mathrm{~mol})$ in ethanol $(30 \mathrm{~mL})$ and few drops of piperidine was refluxed for $3 \mathrm{~h}$, The resulting solid after cooling was collected and recrystallized from the proper solvent to give 4a-c (70-80\% yield);. 4a: IR: 2,208, 2,200 (2 CN): ${ }^{1} \mathrm{H}-\mathrm{NMR}: 10.15$ (s, 1H, CH=C), 8.65 (s, 1H, H-5), 8.55-7.52 (m, 12H, Ar-H). 4b: IR: 3,464, 3,368 $\left(\mathrm{NH}_{2}\right), 2,210(\mathrm{CN}), 1,698(\mathrm{CO}):{ }^{1} \mathrm{H}-\mathrm{NMR}: 9.19(\mathrm{~s}, 1 \mathrm{H}, \mathrm{CH}=\mathrm{C}), 8.60(\mathrm{~s}, 1 \mathrm{H}, \mathrm{H}-5), 8.55-7.52(\mathrm{~m}$, 14H, $\left.\mathrm{Ar}-\mathrm{H}+\mathrm{NH}_{2}\right)$. 4c: IR: 3,344, 3,286 $\left(\mathrm{NH}_{2}\right), 2,212(\mathrm{CN}), 1,340(\mathrm{C}=\mathrm{S}):{ }^{1} \mathrm{H}-\mathrm{NMR}: 10.12$ (br, 2H, $\mathrm{NH}_{2}$ ), 9.67 (s, 1H, $\left.\mathrm{CH}=\mathrm{C}\right), 9.19$ (s, 1H, H-5), 8.59-7.48 (m, 12H, Ar-H).

Synthesis of 5-((3-(naphtho[2,1-b]furan-2-yl)-1-phenyl-1H-pyrazol-4-yl) methylene) pyrimidine2,4,6(1H,3H,5H)trione (5): A mixture of aldehyde $3(3.38 \mathrm{~g}, 0.01 \mathrm{~mol})$ and barbituric acid (0.01 mol) in ethanol $(30 \mathrm{~mL})$ containing triethylamine $(0.01 \mathrm{~mL})$ was heated under reflux for $1 \mathrm{~h}$. The solid separated on heating was filtered off and recrystallized from ethanol to give 5 ( $80 \%$ yield). IR: 3,200 $(\mathrm{NH}), 1,700$ (CO), 1,670 (CO): ${ }^{1} \mathrm{H}-\mathrm{NMR}: 11.38,11.30$ (2s, 2H, 2NH), 9.67 (s, 1H, CH=C), 8.77 (s, 1H, H-5), 8.27-7.36 (m, 12H, Ar-H).

Synthesis of 1-((3-(naphtho[2,1-b]furan-2-yl)-1-phenyl-1H-pyrazol-4-yl)methylene) hydrazone (6a), 1((3-(naphtho[2,1-b] furan-2-yl)-1-phenyl-1H-pyrazol-4-yl) methylene)-2-phenylhydrazone (6b), $N$ '-((3(naphtho[2,1-b]furan-2-yl)-1-phenyl-1H-pyrazol-4-yl)methylene) acetohydrazone (6c), 1-((3(naphtha[2,1-b]furan-2-yl)-1-phenyl-1H-pyrazol-4-yl)methylene) thiosemicarbazone (6d) and 2cyano-N'-((3-(naphtho[2,1-b] furan-2-yl)-1-phenyl-1H-pyrazol-4-yl)methylene)acetohydrazone (6e): A mixture of aldehyde $3(3.38 \mathrm{~g}, 0.01 \mathrm{~mol})$ and hydrazine hydrate, phenyl hydrazine, acetylhydrazine, thiosemicarbazide or cyanoacetic acid hydrazide $(0.01 \mathrm{~mol})$ in ethanol $(30 \mathrm{~mL})$ was refluxed for $3 \mathrm{~h}$, the solid separated on heating was filtered off and recrystallized from a suitable solvent to give 6a-e (75-80 \%). 6a: IR: 3,400, 3,364 $\left(\mathrm{NH}_{2}\right), 1,598(\mathrm{C}=\mathrm{N}),{ }^{1} \mathrm{H}-\mathrm{NMR}: 8.78(\mathrm{~s}, 1 \mathrm{H}, \mathrm{CH}=\mathrm{N}), 8.41(\mathrm{~s}, 1 \mathrm{H}, \mathrm{H}-5)$, 8.37-7.33 (m, 12H, Ar-H), 6.86 (brs, 2H, $\left.\mathrm{NH}_{2}\right)$. 6b: IR: 3,320 (NH), 1,602 (C=N); ${ }^{1} \mathrm{H}-\mathrm{NMR}: 10.39$ (brs, 1H, NH), $9.01(\mathrm{~s}, 1 \mathrm{H}, \mathrm{CH}=\mathrm{N}), 8.40(\mathrm{~s}, 1 \mathrm{H}, \mathrm{H}-5), 8.36-7.17(\mathrm{~m}, 12 \mathrm{H}, \mathrm{Ar}-\mathrm{H}) .6 \mathrm{c}:$ IR: 3,156 (NH), 1,686 (CO); ${ }^{1} \mathrm{H}-\mathrm{NMR}: 11.60$ (brs, 1H, NH), 11.26 (s, 1H, CH=N), 9.17 (s, 1H, H-5), 9.06-7.34 (m, 12H, Ar-H), 2.26 (s, 3H, Me). 6d: IR: 3,420, 3,374, 3,262, 3,178 (NH, NH$), 1,334(\mathrm{C}=\mathrm{S})$; ${ }^{1} \mathrm{H}-\mathrm{NMR}$ : 
11.40 (s, 1H, NH), $9.27(\mathrm{~s}, 1 \mathrm{H}, \mathrm{CH}=\mathrm{N}), 8.72$ (s, 1H, H-5), 8.32 (brs, 2H, NH$)_{2}, 8.41-7.41(\mathrm{~m}, 12 \mathrm{H}$, Ar-H). 6e: IR: 3,310, 3,210, (NH), 2,256 (CN), 1,666 (CO); ${ }^{1} \mathrm{H}-\mathrm{NMR}: 12.02$ (brs, 1H, NH), 9.10 (s, $1 \mathrm{H}, \mathrm{CH}=\mathrm{N}), 8.9$ (s, 1H, CH-pyrazole), 8.93-7.41 (m, 12H, Ar-H), 4.28 (s, 2H, $\left.\mathrm{CH}_{2}\right)$.

Synthesis of N-((3-(naphtho[2,1-b]furan-2-yl)-1-phenyl-1H-pyrazol-4-yl)methylene) aniline/4-chloroaniline (7a,b): A mixture of aldehyde $3(3.38 \mathrm{~g}, 0.01 \mathrm{~mol})$ and the appropriate aromatic amine, namely aniline or $p$-chloroaniline $(0.01 \mathrm{~mol})$ in ethanol $(40 \mathrm{~mL})$ was refluxed for $1 \mathrm{~h}$. The obtained product was collected and recrystallized from the proper solvent to give 7a,b. (80-85\% yield)) 7a: IR: 1,614 $(\mathrm{C}=\mathrm{N})$; ${ }^{1} \mathrm{H}-\mathrm{NMR}: 9.40$ (s, 1H, CH=N), 8.96 (s, 1H, H-5), 8.33-7.36 (m, 17H, Ar-H). 7b: IR: 1,620 $(\mathrm{C}=\mathrm{N}) ;{ }^{1} \mathrm{H}-\mathrm{NMR}: 9.25$ (s, 1H, CH=N), 8.95 (s, 1H, H-5), 8.36-7.35 (m, 16H, Ar-H).

Synthesis of 1-(naphtho[2,1-b]furan-2-yl)-3-(3-(naphtho[2,1-b]furan-2-yl)-1-phenyl-1H-pyrazol-4yl)prop-2-en-1-one (8): A mixture of 2-acetyl naphtho[2,1-b]furan (1, $0.01 \mathrm{~mol})$ with aldehyde 3 (0.01 $\mathrm{mol})$ in ethanol $(30 \mathrm{~mL})$ and few drops of piperidine was heated under reflux for $1 \mathrm{~h}$. The solid separated on heating was filtered off and recrystallized from dioxane to give 8 (80 \% yield): IR: 1,662 (CO), ${ }^{1} \mathrm{H}-\mathrm{NMR}: 8.96$ (s, 1H, H-5), 8.30-7.20 (m, 19H, Ar-H), 5.93-5.69(dd, 2H, CH=CH, J=11.4 Hz).

Synthesis of 4-[3-(naphtho[2,1-b] furan-2-yl)-1-phenyl-1H-pyrazol-4-yl]methylene-3-methyl-1-phenyl2-pyrazoline-5-one (10): To a mixture of $4 \mathbf{a}(0.01 \mathrm{~mol})$ and 3-methyl-1-phenyl-2-pyrazolin-5-one $(0.01 \mathrm{~mol})$ in ethanol $(30 \mathrm{~mL})$, triethylamine $(0.01 \mathrm{~mL})$ was added. The resulting mixture was heated under reflux for $30 \mathrm{~min}$ and left to cool. The precipitated product was collected and recrystallized from acetic acid to give 10 (85\%). IR: 1,678 (CO), 1,610 (C=N); ${ }^{1} \mathrm{H}-\mathrm{NMR}: 10.09$ (s, 1H, CH=C), 8.41 (s, $1 \mathrm{H}, \mathrm{H}-5), 8.14-7.18$ (m, 17H, Ar-H), 2.3 (s, 3H, $\left.\mathrm{CH}_{3}\right)$.

Synthesis of ethyl 6-amino-5-cyano-2-methyl-4-(3-(naphtho[2,1-b]furan-2-yl)-1-phenyl-1H-pyrazol-4yl)-4H-pyran-3-carboxylate (12): To a solution of $\mathbf{4 a}(0.01 \mathrm{~mol})$ and ethyl acetoacetate $(0.01 \mathrm{~mol})$ in methylene chloride $(50 \mathrm{~mL})$, triethylamine $(0.01 \mathrm{~mL})$ was added. The mixture was heated under reflux for $4 \mathrm{~h}$, concentrated and allowed to cool. The precipitated product was collected and recrystallized from ethanol to give 12 (70 \% yield). IR: 3,402, 3,328, 3,206 $\left(\mathrm{NH}_{2}\right), 2,194(\mathrm{CN}), 1,690(\mathrm{CO}) ;{ }^{1} \mathrm{H}-$ NMR: 8.58 (s, 1H, H-5), 8.43-7.34 (m, 12H, Ar-H), 6.94 (brs, 2H, $\mathrm{NH}_{2}$, exchangeable by $\mathrm{D}_{2} \mathrm{O}$ ), 5.05 (s, 1H, 4H-pyran), 3.89 (q, 2H, $\mathrm{CH}_{2} ; J=7.0 \mathrm{~Hz}$ ), 2.27 (s, 3H, Me), 0.84 (t, 3H CH $3 ; J=7.0 \mathrm{~Hz}$ ).

Synthesis of 3-amino-1,5-dihydro-1-(3-(naphtho[2,1-b]furan-2-yl)-1-phenyl-1H-pyrazol-4-yl)-5-oxopyrano[2,3-c]chromene-2-carbonitrile (13): To a solution of $4 \mathbf{4 a}(0.01 \mathrm{~mol})$ and 4-hydroxycoumarin $(0.01 \mathrm{~mol})$ in ethanol $(50 \mathrm{~mL})$ a few drops of piperidine $(0.01 \mathrm{~mL})$ were added. The mixture was heated under reflux for $4 \mathrm{~h}$ and allowed to cool. The precipitated product was collected and recrystallized from dioxane to give 13 (70\% yield). IR: 3,406, 3,324, 3,294 $\left(\mathrm{NH}_{2}\right), 2,196$ (CN), 1,708 (CO); ${ }^{1} \mathrm{H}-\mathrm{NMR}$ : 8.74 (s, 1H, H-5), 8.38-7.41 (m, 18H, Ar-H + NH 2 ), 5.06 (s, 1H, 4H-pyran).

Synthesis of 2-[(3-naphtho[2,1-b]furan-2-yl)-1-phenyl-1H-pyrazol-4-yl]methylene-hydrazonothiazolidin-4-one (14) and 2-[(3-Naphtho[2,1-b]furan-2-yl)-1-phenyl-1H-pyrazol-4-yl]methylenehydrazono-4-methylthizole (15). A mixture of $\mathbf{6 d}(0.01 \mathrm{~mol})$, ethyl bromoacetate $(0.01 \mathrm{~mol})$ or chloroacetone $(0.01 \mathrm{~mol})$ and fused sodium acetate $(0.02 \mathrm{~mol})$ in ethanol $(40 \mathrm{~mL})$ was refluxed for $2 \mathrm{~h}$. 
The product obtained was collected by filtration, washed with water and recrystallized from acetic acid to give 14, 15. Compound 14: 80\% yield; IR: 3,400, 3,300 (NH), 1,706 (CO); ${ }^{1} \mathrm{H}-\mathrm{NMR}$ : 11.51 (brs, $1 \mathrm{H}, \mathrm{NH}), 9.28(\mathrm{~s}, 1 \mathrm{H}, \mathrm{CH}=\mathrm{N}), 8.78(\mathrm{~s}, 1 \mathrm{H}, \mathrm{H}-5), 8.71-7.42(\mathrm{~m}, 12 \mathrm{H}, \mathrm{Ar}-\mathrm{H}), 4.00\left(\mathrm{~s}, 2 \mathrm{H}, \mathrm{CH}_{2}\right)$. Compound 15, 80\% yield; IR: 3,152 (NH), 1,614 (C=N); ${ }^{1} \mathrm{H}-\mathrm{NMR}$ : 11.81 (brs, 1H, NH; exchanged by $\left.\mathrm{D}_{2} \mathrm{O}\right), 9.00(\mathrm{~s}, 1 \mathrm{H}, \mathrm{CH}=\mathrm{N}), 8.51$ (s, 1H, H-5), 8.41-7.39 (m, 12H, Ar-H), 6.40 (s, 1H, CH-thiazoline), $2.19(\mathrm{~s}, 3 \mathrm{H}, \mathrm{Me})$.

Synthesis of $\mathrm{N}$-(4-acetyl-4,5-dihydro-5-(3-(naphtho[2,1-b]furan-2-yl)-1-phenyl-1H-pyrazol-4-yl)1,3,4-thiadiazol-2-yl)acetamide (16): A solution of $\mathbf{6 d}(0.01 \mathrm{~mol})$, and acetic anhydride $(5 \mathrm{~mL})$ was heated under refluxed for $3 \mathrm{~h}$. After the reaction mixture had attained room temperature, excess acetic anhydride was decomposed by water $(10 \mathrm{~mL})$ and the mixture was stirred for $30 \mathrm{~min}$. The separated product was filtered and recrystallized from acetic acid to give 16 (60\% yield); IR: 3,248 (NH), 1,664, 1,634 (2CO); ${ }^{1} \mathrm{H}-\mathrm{NMR}: 11.78$ (brs, 1H, NH), 8.47 (s, 1H, H-5), 8.30-7.35 (m, 12H, Ar-H), 6.44 (s, $1 \mathrm{H}, \mathrm{CH}$-thiapyrazole) 2.31 (s, 3H, Me), 2.01 (s, 3H, Me).

\section{Antibacterial activity}

The newly synthesized compounds were screened for their antimicrobial activities in vitro against two species of Gram-positive bacteria Bacillus subtilis (NCTC- 10400)(BS), Staphylococcus aureus (NCTC 7447)(SA), and three Gram-negative bacteria, Escherichia coli (NCTC 10410)(EC), Pseudomonas aeruginosa (ATCC 10415)(PA), Candida albicans (IMRU 3669)(CA), and one fungus, Aspergillus niger (ATCC 6275)(AN). The activities of these compounds were tested using the disc diffusion method $[19,20]$. The area of zone of inhibition was measured using neomycin (30 $\mu \mathrm{g} \mathrm{mL}^{-1}$ ) as standard antibiotic (Table III). The tested compounds were dissolved in $N, N$ dimethylformamide (DMF) to give a solution of $1 \mathrm{mg} \mathrm{mL}^{-1}$. The inhibition zones were measured in millimeters at the end of an incubation period of $48 \mathrm{~h}$ at $28{ }^{\circ} \mathrm{C} . N, N$-dimethylformamide (DMF) showed no inhibition zone.

\section{Conclusions}

A series of novel naphtho[2,1-b]furan pyrazole derivatives were prepared. The antimicrobial activity of these compounds was evaluated against various Gram-positive, Gram-negative bacteria and fungi. 2-[(3-Naphtho[2,1-b]furan-2-yl)-1-phenyl-1H-pyrazol-4-yl]methylenehydrazonothiazolidin-4one (14) showed the highest antibacterial activity, while compound 2 showed moderate activity, and the remaining tested compounds $\mathbf{4 a - c , ~ 5 , ~ 6 , ~ 7 a , b , ~ 8 a - c , ~ 1 0 , ~ 1 2 , ~} 13$ showed no activities against any of the test microorganisms.

\section{References}

1. Abd El-Wahab, F.H.A; Ali, M.F; El-Agrody, M.A; Bedear H.A; Halawa A.A.; El-Sherbiny G.M. Binary Heterocyclic Systems Containing Ethylidine amino Likage:-Synthesis Of Some New Heterocyclic Compounds Bearing Naphtho[2,1,b] furan Moiety. J. Serb. Chem. Soc. 2006, 71, 459-469. 
2. Cavier, R.; Buisson, J.P.; Lemoine, J.; Royer, R. Comparaison des activités antibactériennes et protozoocides de dérivés méthoxylés ou halogénés des nitrobenzofurannes et des nitronaphthofurannes. Eur. J. Med. Chem. 1981, 16, 73-76.

3. Royer, R.; Buisson, J.P. Recherche sur les dérivés nitrés d'intérêt biologique: XX. Modalités de synthèse des dérivés méthoxylés ou halogénés des nitro-2 naphthofurannes. Eur. J. Med. Chem. 1980, 18, 79-84.

4. Arnaise, S.; Boeuf, H.; Buisson, J.P.; Cantat, N.; Demerseman, P.; Einhorn, J.; Lamotte, G.; Lemelin, M.; Brimer, P.A.; Perdue, S.W.; Hsie, A.W.; Royer, R.; Kelly, F.; Hofnung, M. Genotoxic activity of 2-nitronaphthofurans and related molecules. Mutagenesis 1986, 3, 217-229.

5. Quillardet, P.; Touati, E.; Hofnung, M. Influence of the uvr-dependent nucleotide excision repair on DNA adducts formation and mutagenic spectrum of a potent genotoxic agent: 7-Methoxy-2nitronaphtho[2,1-b]furan (R7000). Mutat. Res. 1996, 358, 113-122.

6. Weill-Thévenet, N.; Buisson, J.P.; Royer, R.; Hofnung, M. Genetic toxicology studies with 2nitrobenzofurans and 2-nitronaphthofurans. Mutat. Res. 1982, 104, 1-8.

7. Touati, E.; Krin, E.; Quillardet, P.; Hofnung, M. 7-Methoxy-2-nitronaphtho[2,1-b]furan (R7000)induced mutation spectrum in the lacI gene of Escherichia coli: Influence of SOS mutagenesis. Carcinogenesis 1996, 17, 2543-2550.

8. Salmon, R.J.; Buisson, J.P.; Aussepe, L.; Royer, R. Induction of sarcomas in rats by subcutaneous injection of 7-methoxy-2-nitro-naphtho[2,1-b]furan (R7000). Carcinogenesis 1985, 6, 109-111.

9. Salmon, R.J.; Buisson, J.P.; Vielh, P.; Aussepe, L.; Royer, R. Cancer épidermoïde du rumen induit chez la souris $\mathrm{C} 3 \mathrm{H}$ par le méthoxy-7-nitro-2 naphtho [2,1-b] furanne (R7000). C. R. Soc. Biol. 1986, 180, 622-624.

10. Quillardet, P.; Michel, V.; Arrault, X.; Hofnung, M.; Touati, E. Mutagenic properties of a nitrofuran, 7-methoxy-2-nitro-naphtho[2,1-b]furan (R7000), in lacI transgenic mice. Mutat. Res. 2000, 470, 177-188.

11. Touati, E.; Phillips, D.H.; Buisson, J.P.; Quillardet, P.; Royer, R.; Hofnung, M. DNA adduct formation by 7-methoxy-2-nitro-naphtho[2,1-b]furan (R7000), an extremely potent mutagen. Mutagenesis 1989, 4, 254-258.

12. Touati, E.; Phillips, D.H.; Quillardet, P.; Hofnung, M. Determination of target nucleotides involved in 7-methoxy-2-nitro-naphtho[2,1-b]furan (R7000)-DNA adduct formation. Mutagenesis 1993, 8, 149-154.

13. Weill-Thévenet, N.; Buisson, J.P.; Royer, R.; Hofnung, M. Mutagenic activity of benzofurans and naphthofurans in the Salmonella/microsome assay: 2-Nitro-7-methoxy-naphtho[2,1-b]furan (R7000), a new highly potent mutagenic agent. Mutat. Res. 1981, 88, 355-362.

14. Maurizis, J.C.; Madelmont, J.C.; Parry, D.; Meyniel, G. Disposition in rats and mice of 7methoxy-2-nitronaphtho[2,1-b]furan. Xenobiotica 1985, 15, 141-148.

15. Maurizis, J.C.; Madelmont, J.C.; Parry, D.; Dauzonne, D.; Royer, R.; Chabard, J.L. Main excretion metabolites of 7-methoxy-2-nitronaphtho[2,1-b]furan. Xenobiotica 1986, 16, 635-643.

16. Latha, K.P.; Vaidya, V.P.; Keshvayya, J.; Vijay Kumar, M.L.; Shreedhara, C. Synthesis of 2acetylnaphtho[2,1-b]furan. Nat. Acad. Sci. Lett. 2002, 25, 153.

17. Attaryan, O.; Antanosyan, S.; Panosyan, G.; Asratyan, G.; Matsoyan, S. Vilsmeier formyatiom of 3,5-dimethylpyrazoles. Rus. J. Chem. 2006, 76, 1817-1819. 
18. El-Agrody, A.M. Activated nitriles in hetorcyclic synthesis: Synthesis of several new naphtho [2, 1-b] pyran-3-one derivatives. J. Chem. Res. 1994, 50-51.

19. Carrod, L.P.; Grady, F.D. Antibiotic and Chemotherapy, 3rd ed.; Churchill Livingstone: Edinburgh, UK, 1972; p. 47.

20. Hewitt, W.; Vincent, S. Theory and Application of Microbiological Assay; Academic Press: New York, NY, USA, 1989.

Sample Availability: Samples are available from the authors.

(C) 2011 by the authors; licensee MDPI, Basel, Switzerland. This article is an open access article distributed under the terms and conditions of the Creative Commons Attribution license (http://creativecommons.org/licenses/by/3.0/). 